DOI: $10.24850 /$ j-tyca-2020-05-08

Artículos

\title{
Revisión de sistemas de telemetría en ríos: propuesta para el río Magdalena, Barranquilla, Colombia
}

\section{Review of telemetry systems in rivers: Proposal for the Magdalena River, Barranquilla, Colombia}

Yuleisy Núñez-Blanco1, ORCID: https://orcid.org/0000-0002-5821-3258

Elkin Ramírez-Cerpa2, ORCID: https://orcid.org/0000-0002-6634-5170

Andrés Sánchez-Comas33, ORCID: https://orcid.org/0000-0002-42808070

${ }^{1}$ Departamento de Civil y Ambiental, Universidad de la Costa CUC, Barranquilla, Colombia, ynunez2@cuc.edu.co

2Departamento de Ciencias de la Computación y Electrónica, Universidad de la Costa CUC, Barranquilla, Colombia, eramirez1@cuc.edu.co

3Departamento de Gestión Industrial, Agroindustrial y Operaciones, Universidad de la Costa CUC, Barranquilla, Colombia, asanchez@cuc.edu.co 
Autora para correspondencia: Yuleisy Núñez-Blanco, ynunez2@cuc.edu.co

\section{Resumen}

La aplicación de tecnologías para la caracterización físico-química en ríos es necesaria para estandarizar las metodologías, obtener resultados precisos y valorar el estado ambiental de las aguas superficiales. Actualmente, en Colombia, la toma de muestras de parámetros de calidad de agua en ríos es efectuada por técnicos de campo, para luego realizar el análisis en los laboratorios, lo cual necesita de organización logística, que demanda considerables recursos financieros y tiempo. El río Magdalena es uno de los cuerpos de agua más importantes del país por el alto tráfico marítimo comercial, fuente de potabilización y vertimiento de aguas tratadas; es necesario aplicar tecnologías para la caracterización físico-química y obtener resultados precisos de valoración del estado ambiental de las aguas superficiales de dicho cuerpo de agua. Con el fin de definir una propuesta de arquitectura para un sistema de telemetría de calidad de agua para el río Magdalena, se hizo una revisión bibliográfica, para identificar las variables más importantes de monitoreo de parámetros de calidad de agua en ríos y se relacionaron con tecnologías usadas para tales fines, como, por ejemplo, casos aplicados. 
Palabras clave: telemetría, tecnología, calidad de agua, agua superficial, río Magdalena.

\section{Abstract}

The application of technologies for physical-chemical characterization in rivers is necessary to standardize the methodologies, obtain accurate results and assess the environmental status of surface waters. Currently, in Colombia, the taking of samples of water quality parameters in rivers, is regularly done by field technicians, to then perform the analysis in the laboratories, which needs logistics organization that requires considerable financial resources and time. The Magdalena River, being one of the most important bodies of water in the country due to the high commercial maritime traffic, source of purification and discharge of treated water, begins to be necessary to apply technologies for physical-chemical characterization and obtain precise results to evaluate the environmental status of surface waters in real-time. Aiming to build a proposal for an architecture of a Water Quality Telemetry System for the Magdalena River, a literature review was conducted. The study identified the most important variables to monitor water quality parameters in rivers and related them to the technologies used for such purposes based on applied cases.

Keywords: Telemetry, technology, water quality, surface water, Magdalena River. 
Recibido: 24/04/2019

Aceptado: $23 / 02 / 2020$

\section{Introducción}

Los ríos son un complejo sistema de agua con movimiento natural, constituido a partir de las variaciones climáticas y distintas formaciones geológicas que, por medio de numerosas transformaciones hidrológicas, geomorfológicas y edáficas han generado una gran variedad de ecosistemas que se interrelacionan de manera espacial y temporal (Martínez \& Pinilla, 2017). Son de gran importancia para la evolución de

los pueblos y civilizaciones, y conllevan intereses políticos, sociales y económicos. Pero hoy en día, factores como la diversidad geográfica, el clima y las actividades antrópicas son patrones que han incrementado su vulnerabilidad, propiciando inseguridad en el mundo por las afectaciones que puedan resultar (Aguirre, Piraneque, \& Cruz, 2018). 
Tecnología y

Ciencias $₫$ Agua
2020, Instituto Mexicano de Tecnología del Agua

Open Access bajo la licencia CC BY-NC-SA 4.0

(https://creativecommons.org/licenses/by-nc-sa/4.0/)

Teniendo en cuenta que los ríos brindan diferentes beneficios para las actividades cotidianas, el ser humano se ha visto en la necesidad de usar la tecnología como medio para monitorear el estado en tiempo real de los mismos. Los sensores remotos in situ son unas de las principales tecnologías utilizadas para el control y monitoreo constante del agua, como los sensores de nivel, que permiten alertar sobre posibles inundaciones (Prafanto \& Budiman, 2018); son útiles para investigaciones en fuerzas navales (Raimondi, Trapanese, Franzitta, \& Viola, 2015). En el campo académico, los datos de los sensores se usan para caracterizar el estado del clima de una determinada región mediante el análisis estadístico de éstos (Ramírez-Cerpa, Acosta-Coll, \& Vélez-Zapata, 2017). Por otro lado, también se utiliza la tecnología para verificar la calidad del agua (Ding, Li, Lin, \& Wang, 2016) tanto para certificar sus diferentes usos como para comprobar el cumplimiento de parámetros de calidad en vertimientos hacia los ríos (Liu, Chen, Gao, \& Chen, 2011). De igual forma, sirve para preservar el estado de especies de plantas y animales (Beatty et al., 2018).

El río Magdalena es uno de los más importantes de Colombia. Es fuente de alimento para los pueblos de sus alrededores a través de la pesca, también se aprovecha como medio de transporte y comunicaciones, debido a que es una vía de acceso hacia los lugares adyacentes al mismo; a esto se suma su belleza, que sirve como atractivo para turistas nacionales y extranjeros por sus Ilamativos paisajes (Vilardy, 2015). En el río Magdalena se llevaron a cabo diferentes estudios de calidad de agua para determinar su estado (Restrepo et al., 2015); sin embargo, al revisar la literatura existente, fue evidente que gran parte de 
éstos fueron en laboratorios y pocos los llevados a cabo con sensores remotos. No obstante, en la revisión se encontraron trabajos sobre telemetría en cuerpos de agua similares.

El presente estudio tiene como finalidad proponer una arquitectura para un sistema de telemetría de calidad de agua para el río Magdalena, en el tramo circundante de la ciudad de Barranquilla, Colombia. Para esto se revisó la literatura con base en dos objetivos: el primero, para identificar investigaciones realizadas en ríos en los que hayan empleado tecnologías de sensores remotos, con el propósito de identificartipos de tecnología, así como características y parámetros de medición de interés para tales cuerpos de agua. El segundo objetivo buscó recopilar estudios de calidad de agua sobre este tipo de cuerpos hídricos en el mundo y sobre el río Magdalena, para definir las principales variables de interés.

La investigación desarrollada fue de tipo descriptiva, pues se expresan las características de investigaciones previas, las tecnologías utilizadas y segmentadas en estudios internacionales y colombianos. La revisión sistemática de la literatura se realizó siguiendo la metodología de Sánchez-Comas, Neira, y Cabello (2016). Se consultaron diversas fuentes de información para definir los conceptos clave relacionados con la temática de telemetría en ríos y definir así las cadenas de búsqueda, con las cuales se consultaron bases de datos especializadas de donde se seleccionaron de la literatura documentos de tipo científico, así como memorias publicadas durante seis años (2013-2018) tanto en español como en inglés. Los documentos elegidos debían cumplir con los siguientes criterios de inclusión: 1) exponer algún estudio de parámetros 
de calidad de agua; 2) el estudio de calidad de agua usaba tecnologías y metodologías de monitoreo remoto. Asimismo, se descartaron documentos que tenían algunos de los siguientes criterios de exclusión: 1) propuestas metodológicas o conceptuales de estudios de gestión ambiental del uso de agua; 2) análisis comparativos de rendimiento de tecnologías utilizadas en la medición de calidad de agua, ya fueran de laboratorio o con sistemas de telemetrías.

\section{Estudios internacionales de calidad de agua y telemetría}

Una de las principales necesidades de las comunidades o asentamientos humanos es el agua. Las actividades económicas dependen de este recurso, y uno de los índices que afectan dichas actividades es la calidad del agua. Tavakoly, Monazami, Rezayi, Tajfard y Borgheipour (2018) midieron y realizaron un análisis de multiparámetros físico-químicos, concluyendo que las fuentes antrópicas y los procesos naturales aumentan las concentraciones de nutrientes, afectando la calidad del agua en determinados asentamientos en Malasia, cerca de Kuala Lumpur. Investigaciones de Hatami (2018) incluyeron como parámetros de 
medición in situ los siguientes: salinidad del agua, temperatura, oxígeno disuelto, $\mathrm{pH}$ y turbidez, para determinar un protocolo unificado de entendimiento entre científicos, y los gestores de recursos en cuanto a las causas y efectos de las aguas residuales en los ecosistemas; este desarrollo se realizó basado en la región del norte de Victoria, Australia. Otro trabajo desarrollado sobre la cuenca del río Guayas, en Ecuador (Damanik-Ambarita et al., 2018), relaciona el impacto ecológico y la medición de la calidad de agua con los métodos para cuantificarel impacto del uso del suelo.

El río Mesta es un afluente que recorre Bulgaria y la frontera con Grecia. A pesar de encontrarse relativamente en buen estado, es objeto de estudio (Georgieva, Gartsiyanova, Ivanova, \& Vladimirova, 2018), pues se ubica en un área rodeada de industrias, granjas agrícolas y asentamientos; esta investigación recomienda un listado de acciones de control y prevención de aguas residuales. De igual manera, las actividades antrópicas son objetivo de estudio (Chen \& Lu, 2014), en este caso, para el manejo eficiente de los recursos hídricos para el sistema de monitoreo del río Cao-E, en China.

Por otro lado, en diferentes investigaciones (Alves et al., 2014; Mustapha, Aris, Juahir, Ramli, \& Kura, 2013; Naddeo, Scannapieco, Zarra, \& Belgiorno, 2013; Panda, Sundaray, Rath, Nayak, \& Bhatta, 2006) resalta la preocupación de gobiernos, organizaciones y entidades locales por monitorear el estado de los ríos y estuarios debido a que son uno de los principales recursos hídricos de las ciudades. Se usan diversas técnicas de muestreo en estos estudios y se obtiene una conclusión estadística con 
respecto a la cantidad permitida de cada variable tanto física como química. Dichos estudios posibilitan, en gran manera, regular la calidad del agua cercana en las urbes grandes o pequeñas.

Desde un enfoque tecnológico, la telemetría es un aspecto importante para la aplicación de aquellos métodos de análisis estadísticos que permiten la evaluación de la calidad del agua y la vida acuática. En Grecia, exactamente dentro de un área minera, se realizó un estudio (Sfikas, Angelidis, \& Petridis, 2015) que utiliza un sistema de telemetría con sensores multiparámetros para desarrollar una metodología que identifica incidentes de contaminación potencial. En el río Zarqa de Jordania, debido al crecimiento poblacional en la zona, se usa un sistema de telemetría (Al-Omari, Al-houri, \& Al-Weshah, 2013) que realiza el seguimiento de las aguas residuales tratadas en dos estaciones de monitoreo.

En conjunto con los análisis estadísticos de los datos, estos sistemas de telemetría han sido una herramienta clave para el seguimiento o trazabilidad de diversas especies marinas, y se ha demostrado en distintas investigaciones (Turnure, Grothues, \& Able, 2014; Kraus, Secor, \& Wingate, 2015; Tétard et al., 2016), que primordialmente emplearon técnicas de telemetría acústica pasiva, y además monitorearon otros parámetros relacionados con la calidad del agua, como el oxígeno o la temperatura.

Para los autores, esta investigación se ubica en tales sistemas de telemetría y manejo de datos en favor del monitoreo de la calidad del 
agua. A continuación se dará una muestra de las principales investigaciones encontradas en el ámbito nacional al respecto.

\section{Estudios colombianos de calidad de agua y telemetría}

Los recursos hídricos desempeñan un papel importante en todos los sectores económicos, como agricultura, ganadería, actividades industriales y recreación, entre otras. La disponibilidad y calidad del agua superficial se ha deteriorado debido a factores como la industrialización, aumento demográfico y consumo desenfrenado. La calidad es el reto más importante para la gestión de dicho recurso, pues se clasifica de acuerdo con el grado de contaminación y pureza, además de que se evalúan las características químicas, físicas y biológicas, causando que su evaluación, análisis y distribución sean tareas complejas (Pérez, Nardini, \& Galindo, 2018). Sin embargo, en el ámbito nacional, algunas investigaciones han evaluado distintas alternativas tecnológicas apropiadas para brindar la caracterización precisa de la calidad del agua del país.

Por ejemplo, Velásquez-Villada y Donoso (2016), en el proyecto GOLDFISH-Detección de la contaminación del recurso agua en países en 
desarrollo mediante redes de sensores, concluyeron que las comunicaciones desde áreas remotas pueden ser de interés, pero siguen siendo un problema en Colombia. En este proyecto se trabajó con la plataforma Raspberry Pi para integrar todos sus componentes, y en el desarrollo de tecnología de sensores, módulos de comunicación, plataforma de administración y monitoreo central, así como en algoritmos de comunicación. En la extensión del proyecto se agregó una tecnología de generación de energía y una aplicación de localizador de contaminantes. El proyecto GOLDFISH se diseñó para detectar la contaminación del río con algunos sensores integra dos y para enviar estos datos a un Gateway (GW) en la orilla del río. El GW envía los datos del sensor a la estación central de administración y monitoreo (MMS), a través de una VPN por una red móvil. La comunicación entre los nodos en el río y la GW se logró por medio del estándar IEEE 802.11b Wi-Fi, con una antena flotante, incorporada en el diseño del nodo del sensor. El GW tenía capacidades de comunicación 2G/3G y envió los datos del sensor a través de canales. Una vez que el MMS tuvo los datos del sensor podría mostrar anomalías o alarmas para niveles de contaminantes que superan lo esperado. El sistema fue probado en Europa y Colombia. Sin embargo, para las pruebas en Colombia, el sistema tuvo fallas de comunicación. La comunicación del GW al MMS no funcionó. Había una antena celular cerca del sitio de prueba, pero el rendimiento de los datos varió entre 20 y 95 kbps, y de 450 ms a 2.12 s para llegar a un servidor en Bogotá DC (100 $\mathrm{km}$ del sitio de prueba). Además, la VPN al MMS tuvo que ser creada con un servidor en Varsovia. Después de dos días de pruebas, esta parte de comunicación del proyecto (GW a MMS) no funcionó. Debido a ello, los 
2020, Instituto Mexicano de Tecnología del Agua

Open Access bajo la licencia CC BY-NC-SA 4.0

(https://creativecommons.org/licenses/by-nc-sa/4.0/)

autores buscaron una solución de conectividad en un sitio remoto, cerca de un río, conectando una red de sensores inalámbricos (WSN, por sus siglas en inglés) para el control de la contaminación. Por lo tanto, se sugirieron las redes tolerantes al retardo.

Además de GOLDFISH, Barreto, Barrera y Benavides (1999) realizaron un estudio multidisciplinario del Golfo de Morrosquillo, entre Punta Rada, y Tolú e Isla Fuerte, en el cual se concluyó que los sensores remotos para hacer cartografía y monitoreos en aguas tropicales son mejores sobre otras técnicas in situ, porque suministran la información de manera rápida, con gran cobertura y acceso en áreas difíciles. Sin embargo, el empleo de sensores remotos para ecosistemas marinos en zonas tropicales presenta algunas limitaciones y desventajas, principalmente con la interface aire-agua, situación que se puede presentar también en ríos.

Por su parte, en el estudio de Ardila-León y Quintero-Delgado (2013) se usaron técnicas de teledetección y sistema de información geográfica para conocer la cantidad de área inundada provocada por el río Soapaga; se empleó una relación con la herramienta Resolution Merge y con ayuda del software Erdas Imagine 2010. Sin embargo, no fue posible conocer el área inundable, porque la topografía de la zona tiene pendientes, lo cual permitió que el agua se secara con rapidez.

En otro ejemplo, Forero-Bernal, Zabala-Parra y Boada-Rodríguez (2017) usaron figuras de Radarsat 2 y Landsat para determinar índice de perturbación antrópica por medio de los siguientes indicadores: demografía, flora y fauna, y variación del cauce. Esto permitió evaluar los 
cambios en el espacio y tiempo en las áreas afectadas a causa de las actividades antrópicas. Se concluyó que dicha cuenca tiene mayor ocurrencia humana en sitios urbanos e industriales, porque es importante contar con una buena planificación y gestión del territorio.

Por su parte, en la cuenca del río Opia, localizado entre los municipios de Tolima, Ibagué, Coello y Piedras, Oviedo-Machado y Reinoso-Flórez (2018) estudiaron 19 puntos, que fueron georreferenciados con el GPS Garmin etrex. Estos puntos se categorizaron según tramos equilibrados (sitios sin o con baja actividad humana, protegidos), y tramos desequilibrados o contaminados (sitios afectados por actividades agropecuarias o vertimiento de aguas residuales, residuos u otras actividades). En ellos, se tomaron muestras in situ y se determinó la temperatura y conductividad del agua con un equipo portátil (Schott Handylab multi12/Set). Por último, se concluyó que la composición de la biota en el río se altera por factores ambientales, calidad del agua y su movimiento.

Otro caso es el de Gómez y Dalence (2014). Este estudio fue más específico, utilizó los sólidos suspendidos totales (SST) como variable para la caracterización del agua, haciendo uso de una imagen de resolución de 1.6 metros, multiespectral del satélite GeoEye. A partir del modelo, resultó que la imagen de satélite GeoEye es adecuada para la estimación del parámetro SST, utilizando bandas del espectro visible e infrarrojo cercano, donde se obtuvo un coeficiente de correlación y determinación con valor de 0.9. Sin embargo, al ser un modelo empírico, es aplicable sólo a la zona de estudio, donde es necesario que se 
2020, Instituto Mexicano de Tecnología del Agua

Open Access bajo la licencia CC BY-NC-SA 4.0

(https://creativecommons.org/licenses/by-nc-sa/4.0/)

profundice en la investigación de los límites permisibles del modelo (inferior y superior) para poder aplicarlo de manera confiable.

Por su parte, Caicedo-Carrascal (2008), con ayuda de técnicas de teledetección, estima variables meteorológicas para aplicar y mejorar simulación a un cuerpo de agua. Para ello, usó datos medidos por satélites, porque ofrecen mejor rendimiento. Se tomó el pronóstico de las afluencias al embalse de Betania en el departamento del Huila, con puntos de control en las estaciones hidrológicas de Paicol y Puente Balseadero. Se empleó un modelo matemático aglutinado, las Combinaciones Lineales Adaptativamente Óptimas-CLAO y el modelo distribuido MIKE SHE, que integra en su estructura los diferentes procesos del ciclo hidrológico.

En Barranquilla, Colombia, Cama-Pinto et al. (2016) usaron tecnología en un cuerpo de agua del río Magdalena. Básicamente muestran el diseño de una estructura de red de sensores inalámbricos o WSN (Wireless Sensor Network) para monitorear en tiempo real variables atmosféricas, las cuales ayudan para desarrollar alertas de posibles inundaciones repentinas o, conocidos coloquialmente, "arroyos", a causa de las precipitaciones en un corto tiempo. Para el diseño de la estructura se valoró el área de estudio y también se desarrolló una aplicación web móvil que utiliza el lenguaje unificado de modelado (UML), que muestra con exactitud y en tiempo real un mapa de las calles de la ciudad, y la categorización del arroyo en diferentes tramos por donde curse. 


\section{Parámetros de la calidad del agua}

El análisis de las variables físicas para la calidad de agua en ríos es de gran importancia para mitigar los impactos de contaminación de dicho ecosistema. Los nuevos sistemas de industrialización, producción y consumo, provenientes de actividades como minería, ganadería y actividades recreativas, entre otras, influyen con gran impacto sobre los efluentes. La calidad del agua ha sido clasificada históricamente a través de la evaluación de variables físicas, como transparencia y nutrientes. Además, el enriquecimiento de nutrientes puede conducir al desarrollo de eutrofización, proliferación de algas tóxicas, y síntomas adversos o secundarios. Estos síntomas adversos tienen efectos negativos en los ecosistemas acuáticos, como hipoxia, agotamiento de oxígeno, turbidez y, en última instancia, causan alta mortalidad de organismos acuáticos (Hatami, 2018).

Por lo tanto, en los últimos años se hicieron investigaciones en ríos donde se tiene en cuenta el comportamiento de parámetros en tiempo real que indiquen la calidad del agua, en especial por su aspecto biológico y físico, con el fin de tomar decisiones estratégicas para contribuir a la gestión ambiental. Esto se lleva a cabo por medio de métodos analíticos, que tienen grandes ventajas. 
2020, Instituto Mexicano de Tecnología del Agua

Open Access bajo la licencia CC BY-NC-SA 4.0

(https://creativecommons.org/licenses/by-nc-sa/4.0/)

Los indicadores biológicos se implementaron para examinar el impacto de los contaminantes antrópicos. Éstos muestran la capacidad de los factores estresantes para causar efectos adversos en el medio ambiente, además de que responden a las presiones ambientales, cambiando su abundancia, diversidad y estructuras histológicas (Tavakoly et al., 2018).

Los índices físicos son casi exactos, fáciles de diferenciar, verificar y correlacionar. Sin embargo, sus resultados producen datos específicos y de corto alcance temporal (Martínez \& Pinilla, 2017).

La toma de muestra en campo demanda tiempo, gastos económicos, al igual que las dificultades logísticas que conlleva trasladarse a zonas de difícil acceso, entre otros factores; esto limita la posibilidad de tomar datos y realizar el monitoreo para conocer el comportamiento de los cuerpos de agua en una determinada región. Por tanto, a partir de los sensores remotos es posible medir la radiación solar reflejada por los cuerpos de agua en varias longitudes de onda, lo que permite correlacionar esta información con algunos de los parámetros de calidad de agua tradicionalmente medidos. Lo anterior es posible debido a las propiedades ópticas o de reflectancia del agua, que dependen de la concentración y características de los sedimentos suspendidos, la materia orgánica disuelta y el fitoplancton existente en ésta (Gómez \& Dalence, 2014).

Un ejemplo se observa en el estudio de Sfikas et al. (2015), donde se realizó una medición continua de la calidad del agua utilizando una unidad de sensor multiparamétrico (Watertool II-Terramentor, EEIG). 
2020, Instituto Mexicano de Tecnología del Agua

Open Access bajo la licencia CC BY-NC-SA 4.0

(https://creativecommons.org/licenses/by-nc-sa/4.0/)

Este sistema comprende una sonda que contiene elementos de medida (electrodos y sensores), protegidos por una robusta carcasa metálica cilíndrica. El sistema incluye componentes adicionales para la recolección de datos y transferencia, incluyendo digitalización y control/almacenamiento, así como una unidad de transferencia de datos. Tal dispositivo puede ser utilizado para la medición simultánea de hasta 15 parámetros fisicoquímicos. En este estudio se monitorearon los siguientes seis parámetros: temperatura, conductividad, $\mathrm{pH}$, iones nitrato $\left(\mathrm{NO}_{3}\right)$, iones de cloruro $\left(\mathrm{Cl}^{-}\right)$e iones sulfato $\left(\mathrm{SO}_{2}^{-4}\right)$. Estos parámetros fisicoquímicos particulares se consideraron como representativos de las características de la calidad del agua.

Por su parte, para desarrollar información sobre las condiciones del hábitat se realizó un muestreo en la bahía de Chesapeake, EUA. En cada evento de prospección de telemetría se registraron perfiles verticales de temperatura del agua, salinidad y oxígeno disuelto, utilizando una sonda de parámetros múltiples marca YSI, modelo V6290-V2, con sensor óptico y barómetro interno en cada intersección de la rejilla. Los datos de calidad del agua se resumieron en intervalos de medio metro para los análisis posteriores, que arrojaron $n=6194$ mediciones de la calidad del agua resueltas en profundidad (Kraus et al., 2015).

Para adquirir una distribución detallada de la calidad del agua de los accidentes de contaminación de la superficie de la misma, Ma, Meng, Zhou, Wang y Shi (2018) estudiaron varios nodos de sensores para monitorearla; contenedores no tripulados y estaciones de servicio equipadas con múltiples sensores de calidad del agua, incluidos los 
2020, Instituto Mexicano de Tecnología del Agua

Open Access bajo la licencia CC BY-NC-SA 4.0

(https://creativecommons.org/licenses/by-nc-sa/4.0/)

sensores de espectrometría UV-visible; sensores de conductividad; $\mathrm{pH}, \mathrm{y}$ oxígeno disuelto. En cada punto de monitoreo se utilizó una unidad de muestreo para transferir automáticamente muestras de agua desde el agua de la superficie a su recinto exterior.

En la Tabla 1 se muestran los parámetros físicos que de acuerdo con el Plan Hídrico Nacional de Colombia deben monitorearse y llevarse a cabo vía remota, con el fin de conocer las condiciones reales del recurso agua y llevar un control de la misma. Cada parámetro establece su concepto e importancia descrita por el Instituto de Hidrología, Meteorología y Estudios Ambientales (IDEAM).

Tabla 1. Parámetros de calidad de agua (IDEAM, 2014).

\begin{tabular}{|l|l|}
\hline \multicolumn{1}{|c|}{ Parámetro } & \multicolumn{1}{|c|}{ Concepto } \\
\hline Temperatura & $\begin{array}{l}\text { Las lecturas de temperatura se utilizan en distintos } \\
\text { estudios, como el cálculo de alcalinidad, calcio, } \\
\text { salinidad y saturación, y en diversas experiencias de } \\
\text { laboratorio. En estudios del clima, cuerpos de agua y } \\
\text { calidad de aire, las temperaturas a menudo se } \\
\text { requieren. Ya que es una variable que puede tener } \\
\text { gran impacto ecológico }\end{array}$ \\
\hline Conductividad & $\begin{array}{l}\text { La conductividad es una característica propia del agua } \\
\text { y otras sustancias líquidas para conocer la capacidad } \\
\text { de conducir la corriente eléctrica. Este dominio }\end{array}$ \\
\hline
\end{tabular}


2020, Instituto Mexicano de Tecnología del Agua

\begin{tabular}{|c|c|}
\hline & $\begin{array}{l}\text { depende de la concentración, contenido de iones, } \\
\text { temperatura, movimiento y valencia de los electrones }\end{array}$ \\
\hline Oxígeno disuelto & $\begin{array}{l}\text { El oxígeno disuelto (OD) es esencial para que los } \\
\text { microorganismos aerobios puedan respirar; cabe } \\
\text { resaltar que el oxígeno es poco soluble en el agua, por } \\
\text { tanto puede estar presente en un gas soluble, en la } \\
\text { presión ejercida en la atmósfera, en la pureza y } \\
\text { temperatura del agua. La concentración de OD en } \\
\text { afluentes naturales depende de la demanda } \\
\text { bioquímica de los organismos y de las propiedades } \\
\text { fisicoquímicas del mismo }\end{array}$ \\
\hline $\mathrm{pH}$ & $\begin{array}{l}\text { El pH es la concentración de ión hidrógeno. Se usa } \\
\text { para conocer la magnitud alcalina o ácida de una } \\
\text { solución. Se analiza la actividad potenciométrica de los } \\
\text { iones hidrógeno por el uso de un electrodo de } \\
\text { referencia o de vidrio, o combinado }\end{array}$ \\
\hline Turbidez & $\begin{array}{l}\text { La turbiedad en el agua es causada por suspensión de } \\
\text { materia como sedimentos, arenas, materia orgánica e } \\
\text { inorgánica, y microorganismos. Su propiedad se basa } \\
\text { en la óptica que causa la luz al ser dispersada y } \\
\text { absorbida en un flujo. A mayor turbiedad, mayor } \\
\text { dispersión de la luz. El tamaño, forma e índice de las } \\
\text { partículas afecta las propiedades de dispersión de la } \\
\text { luz de la suspensión y esto es difícil de relacionar }\end{array}$ \\
\hline Alcalinidad & $\begin{array}{l}\text { La alcalinidad en el agua pretende equilibrar los } \\
\text { ácidos, sumando las bases; el dato medido puede } \\
\text { variar con respecto al pH }\end{array}$ \\
\hline
\end{tabular}


2020, Instituto Mexicano de Tecnología del Agua

Open Access bajo la licencia CC BY-NC-SA 4.0

(https://creativecommons.org/licenses/by-nc-sa/4.0/)

\begin{tabular}{|l|l|}
\hline Sólidos & Las aguas superficiales contienen distintos tipos de \\
totales & sólidos: suspendidos, disueltos y sedimentables; \\
& globalmente causan diferentes problemas de color, \\
& $\begin{array}{l}\text { olor, sabor y salud, a menos que sean extraídos } \\
\text { mediante métodos químicos y físicos }\end{array}$ \\
\hline
\end{tabular}

\section{Caracterización del río Magdalena}

El río Magdalena es un complejo sistema de agua con movimiento natural, constituido a partir de las variaciones climáticas y las distintas formaciones geológicas. Por medio de numerosas transformaciones hidrológicas, geomorfológicas y edáficas se ha generado una gran variedad de ecosistemas que se interrelacionan en el tiempo y en el espacio, principalmente por el agua que transcurre desde su nacimiento hasta la desembocadura. Gracias a su profundidad, el río Magdalena es reconocido como una macrounidad territorial. Comprende $24 \%$ del territorio del país; $77 \%$ de los colombianos viven en las zonas aledañas al río y en la que se desarrollan las principales actividades de la economía (Vilardy, 2015). Es navegable desde Honda hasta su boca en el Mar Caribe, y su principal afluente es el río Cauca; produce cerca del $85 \%$ del 
producto interno bruto nacional (PIB). El río Magdalena es considerado el más importante de Colombia, aunque no es el más largo (Martínez \& Pinilla, 2017). Una de las ciudades cercanas al río es Barranquilla, la capital del Departamento del Atlántico en Colombia.

La ciudad de Barranquilla, Colombia, se localiza en las coordenadas $10^{\circ} 59^{\prime} 16^{\prime \prime}$ de latitud, al norte del ecuador, y en longitud a $74^{\circ} 47^{\prime} 20^{\prime \prime}$ al oeste del meridiano de Greenwich, tomando como referencia la Plaza de la Paz. Como se observa en la Figura 1 y Figura 2, la ciudad se encuentra cerca del río Magdalena, por lo que se toma como punto de referencia en este escrito y tal como se describe en Angulo (2017), tiene una ubicación privilegiada para emplear diversos estudios socioeconómicos.

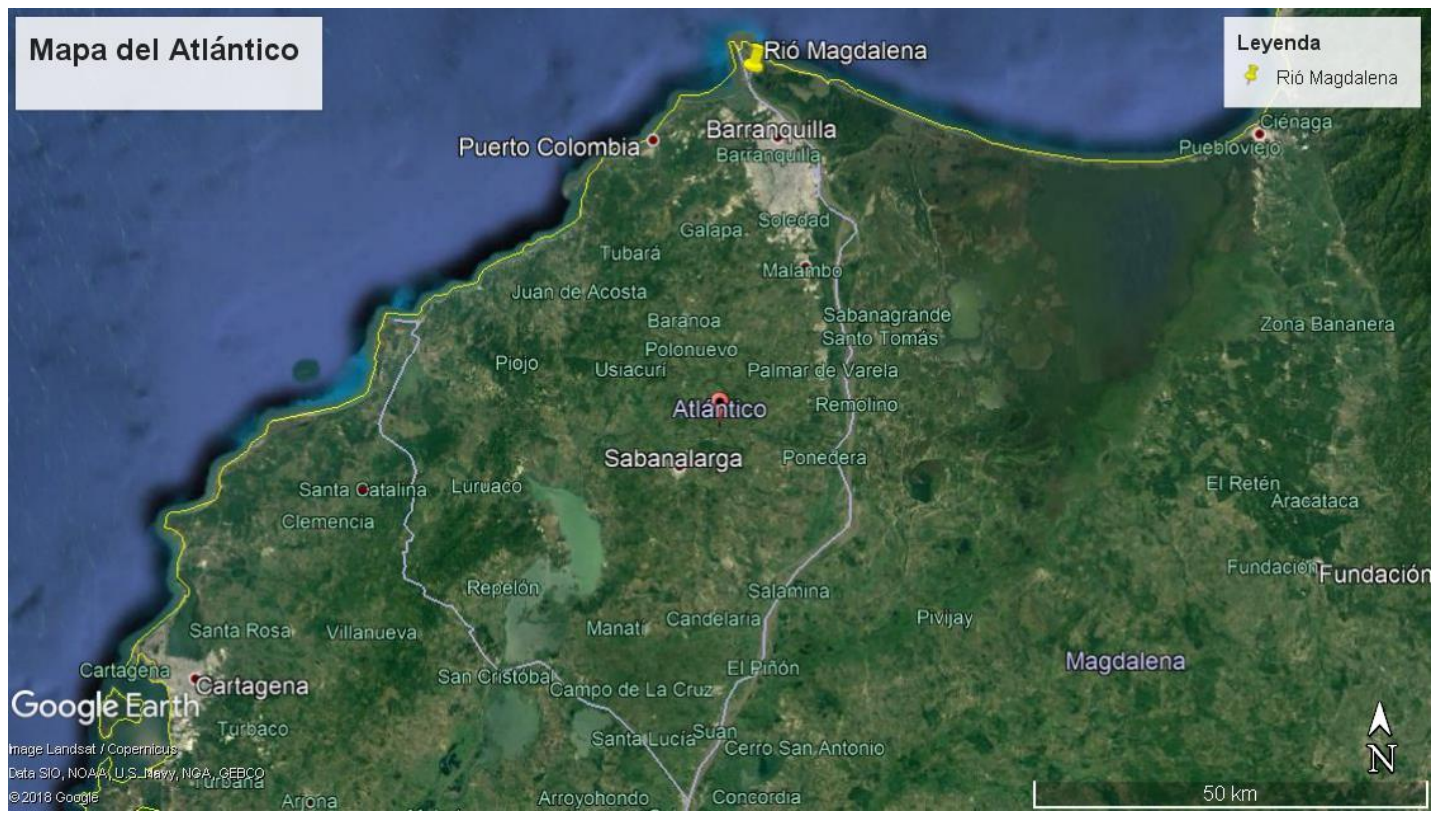

Figura 1. Departamento del Atlántico (Google Maps, s.f., a). 
Tecnología y

Ciencias $\approx$ Agua
2020, Instituto Mexicano de Tecnología del Agua

Open Access bajo la licencia CC BY-NC-SA 4.0

(https://creativecommons.org/licenses/by-nc-sa/4.0/)

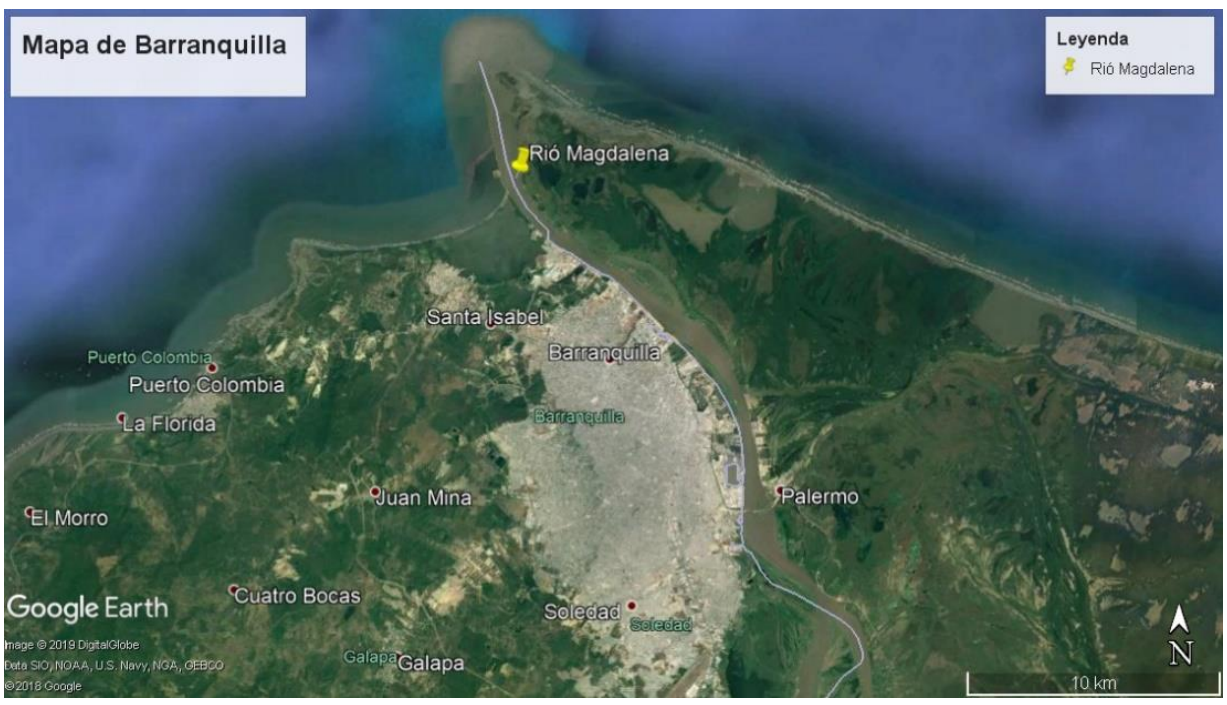

Figura 2. Ciudad de Barranquilla (Google Maps, s.f., b).

El río Magdalena posee las siguientes características: tiene una longitud de $1.528 \mathrm{~km}$, con un caudal de $7095 \mathrm{~m}^{3} / \mathrm{s}^{-1}$. La lámina de agua tiene una pendiente entre $1.2 \times 10^{-5}$ y $6.3 \times 10^{-5}$. El ancho del río varía entre 500 a 750 m, y alcanza una profundidad de 9 metros en promedio. La velocidad del río maneja rangos entre 0.26 a $2.16 \mathrm{~m}^{3} / \mathrm{s}-1$ (Torres, Ramírez-León, Rodríguez, Tejera, \& Vásquez, 2015). La Figura 3 muestra una imagen del río Magdalena. 


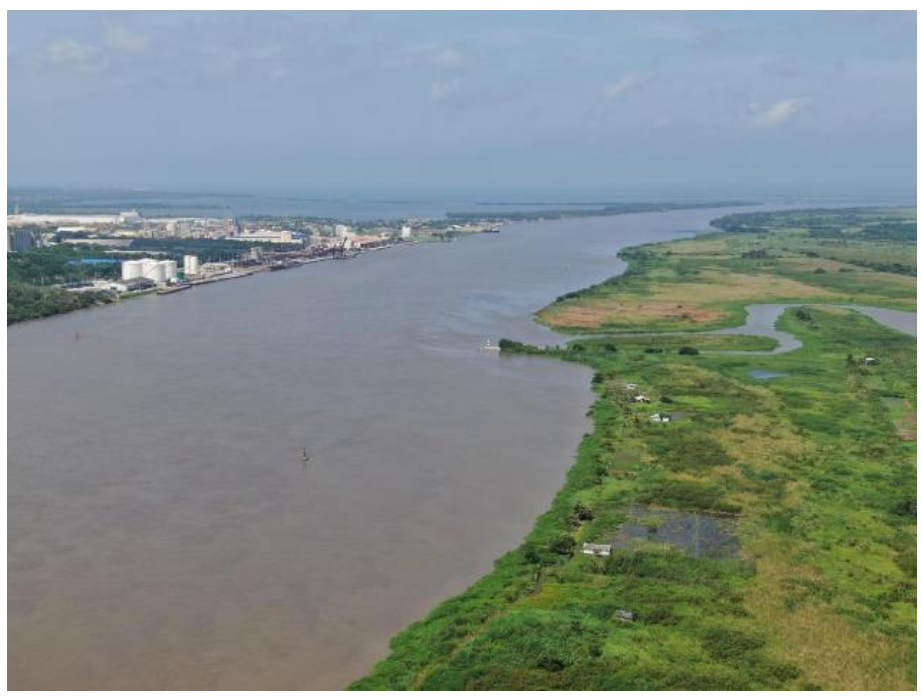

Figura 3. Imagen del río Magdalena, tomada el 20-04-2019 (Barranquilla, Colombia).

El gobierno nacional pretende obtener beneficios económicos para todos los sectores del país, por lo que ha concedido iniciar obras de carácter logístico para que se permita la entrada y salida de mercancía, evitando los problemas de dragado, como se evidencia en la Figura 4. Lo anterior generará dinamismo en el sector, impulsando la creación de nuevos proyectos. 


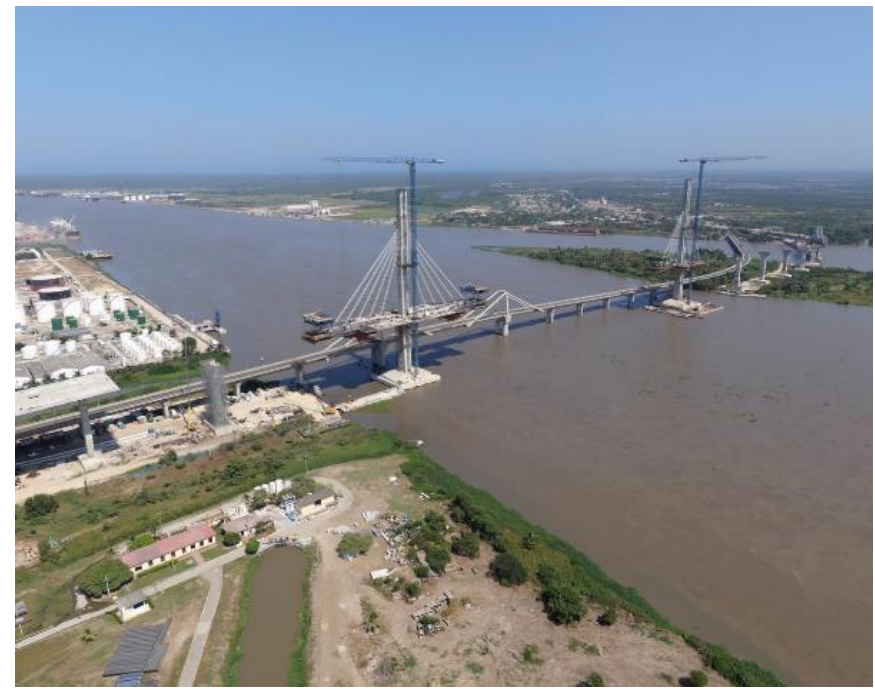

Figura 4. Imagen del puente Pumarejo, tomada el 20-04-2019 (Barranquilla, Colombia).

\section{Tecnologías de telemetría}

Una parte de los estudios tratados en esta revisión utilizan muestras de los ríos o cuerpos de agua, y luego tratan estas muestras en laboratorios especializados. Parte de los parámetros de medición no necesitan traslado hacia los laboratorios; es en este punto donde son útiles los sistemas de telemetría, pues pueden basarse en diversas tecnologías de 
2020, Instituto Mexicano de Tecnología del Agua

Open Access bajo la licencia CC BY-NC-SA 4.0

(https://creativecommons.org/licenses/by-nc-sa/4.0/)

telecomunicaciones, y los más importantes lo hacen en sistemas de radares y redes de sensores inalámbricas (WSN). En este apartado se describirán cómo están conformadas las WSN y las ventajas que ofrecen a partir de la revisión de la literatura.

Una red de sensores se conforma por sensores, nodos sensores, nodo Gateway, estación base e infraestructura de la red inalámbrica establecida para la comunicación entre los componentes (Mahmoud, 2013). Cada uno cumple con una función específica. A partir de esta lectura, es posible conocer algunos sin profundizar en conceptos técnicos.

Una red de sensores realiza lecturas de distintos tipos de sensores que permiten monitorear diversos tipos de parámetros. En el estudio, Pant, Verma y Dhuliya (2017) relacionan una muestra de diferentes sensores y su parámetro de medida. En la Tabla 2 se muestra el resumen.

Tabla 2. Sensores utilizados en telemetría (Pant et al., 2017)

\begin{tabular}{|l|l|l|}
\hline \multicolumn{1}{|c|}{ Parámetro } & \multicolumn{1}{|c|}{ Sensor } & Medida instrumental \\
\hline Temperatura & $\begin{array}{l}\text { Detector de temperatura } \\
\text { resistivo (RTD), termocupla } \\
\text { y termistor }\end{array}$ & Resistencia, voltaje \\
\hline $\begin{array}{l}\text { Turbidez, intensidad } \\
\text { lumínica o señal óptica }\end{array}$ & $\begin{array}{l}\text { Fotodiodo, fototransistor, } \\
\text { sensor infrarrojo, CCD } \\
\text { sensor }\end{array}$ & Corriente \\
\hline Flujo de agua & $\begin{array}{l}\text { Transductores, sensor de } \\
\text { efecto Hall }\end{array}$ & Voltaje, corriente \\
\hline Flujo de aire & Anemómetro & Voltaje, corriente \\
\hline
\end{tabular}


2020, Instituto Mexicano de Tecnología del Agua

Ciencias $\stackrel{\Xi}{\triangleleft}$ gua

Open Access bajo la licencia CC BY-NC-SA 4.0

(https://creativecommons.org/licenses/by-nc-sa/4.0/)

\begin{tabular}{|c|c|c|}
\hline Posición & $\begin{array}{l}\text { Ultrasonido, GPS, infrarrojo, } \\
\text { inclinómetro }\end{array}$ & Voltaje, corriente \\
\hline $\begin{array}{l}\text { Movimiento/aceleración/ } \\
\text { vibración }\end{array}$ & $\begin{array}{l}\text { Acelerómetro/giroscopio/ } \\
\text { sensor fotoeléctrico }\end{array}$ & $\begin{array}{l}\text { Capacitancia, voltaje, } \\
\text { corriente }\end{array}$ \\
\hline Fuerza/presión & $\begin{array}{l}\text { Galga extensiométrica, } \\
\text { piezoeléctrico de cristal, } \\
\text { piezómetro, barómetro, } \\
\text { diafragma capacitivo, celdas } \\
\text { piezo-resistivas, entre otros }\end{array}$ & $\begin{array}{l}\text { Resistencia, voltaje, } \\
\text { corriente }\end{array}$ \\
\hline Humedad & $\begin{array}{l}\text { Sensor de humedad } \\
\text { dieléctrica; en algunos } \\
\text { casos, este sensor está } \\
\text { integrado con el sensor de } \\
\text { temperatura }\end{array}$ & Voltaje, corriente \\
\hline Acústico & $\begin{array}{l}\text { Resonador piezoeléctrico, } \\
\text { micrófono }\end{array}$ & Voltaje, corriente \\
\hline Radiación & $\begin{array}{l}\text { Detector de ionización, } \\
\text { contadores de Geiger- } \\
\text { Mueller }\end{array}$ & Voltaje, corriente \\
\hline Químico & $\begin{array}{l}\text { Sensor de } \mathrm{pH} \text {, sensores } \\
\text { electroquímicos, sensores } \\
\text { de gas infrarrojo }\end{array}$ & Voltaje, corriente \\
\hline
\end{tabular}

Una de las ventajas de utilizar la WSN es conocer el estado de algún parámetro o variable de manera remota, para poder tomar acciones rápidas; por ejemplo, activar alguna alerta de inundación. En un estudio de Singh, Gusain, Mishra, Gupta y Das (2018), utilizaron sensores de temperatura en distintos puntos del río Basin, en India, para analizar 
posibles riesgos de inundación por el deshielo de la nieve en las montañas; otra investigación sobre el río Basin (Zhou et al., 2018), estudió la reducción en tiempo real de las aguas subterráneas de esta cuenca. Khan, Alam, Shahid y Suud (2017) también utilizaron las WSN para prevenir inundaciones y enviar alertas sobre formación huracanes.

Uno de los sectores industriales que está alcanzando un gran auge de las WSN es la acuicultura. Shetty, Pai y Pai (2018) usaron la estructura de una WSN para recopilar información de parámetros del agua que influyen sobre la actividad económica, específicamente al cultivo de algunas especies de plantas y animales de agua cálida. Una aplicación posterior pensada para este trabajo fue extender la aplicación de Internet de las cosas (IoT, por sus siglas en inglés), para mejorar la comodidad del usuario y potenciar las ventajas del monitoreo de los dispositivos. Otra investigación donde se integra WSN con IoT es en la de Alahi, PereiraIshak, Mukhopadhyay y Burkitt (2018), que monitorea la concentración de nitratos en el agua en tiempo real.

La mayoría de estas redes utiliza el enfoque y los protocolos de comunicaciones adoptados en Zigbee, como se menciona en Viswanathan, Sai Shibu, Rao y Ramesh (2017); Guaman, AstudilloSalinas, Vazquez-Rodas, Minchala y Placencia (2018); Samijayani, Sulistya, Wulansari, Mujadin y Rahmatia (2018), y se pueden utilizar en conjunto con otros protocolos, como los adoptados para redes de baja potencia y área amplia (LoRa, por sus siglas en inglés), muy útiles para el uso del IoT. 
En el trabajo de Shen, Sun, Dang, Zou y Wang (2018) se utiliza la WSN como instrumento para el desarrollo de un algoritmo que permite mejorar la vigilancia de niveles de contaminación en ríos y distintos cuerpos de agua.

\section{Sistema de telemetría}

Teniendo en cuenta los estudios descritos en los apartados anteriores, en esta sección se describe una propuesta inicial de los dispositivos que pueden ser utilizados en un sistema de telemetría. Cabe destacar que se tomaron en cuenta las características del río Magdalena ya descritas para elaborar las siguientes métricas:

- Costo: la propuesta del sistema WSN reduce costos en comparación con el uso de los equipos en un laboratorio debido al costo que implica el traslado de muestras, mantenimiento y mano de obra en técnicos para el análisis en los laboratorios. Por otro lado, los sistemas de telemetría de WSN son conocidos en el campo de investigación como sistemas de bajo costo (Jin, Ma, Lv, Lou, \& Wei, 2010; Kamble \& Vatti, 2015), en 
2020, Instituto Mexicano de Tecnología del Agua

Open Access bajo la licencia CC BY-NC-SA 4.0

(https://creativecommons.org/licenses/by-nc-sa/4.0/)

comparación con el costo asociado con el uso de radares meteorológicos ubicados en estaciones meteorológicas gubernamentales.

- Conectividad: el tipo de tecnología de comunicaciones es relevante para saber qué equipos se pueden interconectar al sistema; en otros términos, la frecuencia de operación de los dispositivos.

- Alcance de transmisión: teniendo en cuenta las dimensiones del río, es de vital importancia cubrir la distancia mínima del ancho de éste (500 m).

- Suministro de energía eléctrica: se requiere una fuente de alimentación independiente de la red eléctrica por la ubicación en el río; por ende, es indispensable el uso de paneles solares para tener mayor tiempo de autonomía.

- Escalabilidad: las WSN permiten interconectar gran cantidad de sensores y nodos sensores en cada uno de estos nodos, esto es de gran importancia al momento de expandir la red a más puntos de medición.

- Topología: inicialmente, la WSN puede interconectarse en una red tipo estrella o árbol en caso de que se requiera extender los puntos de medición, es decir, agregar más nodos sensores lejanos del nodo Gateway.

La Figura 5 es un ejemplo de arquitectura de red del sistema de telemetría, que consiste en una red tipo estrella entre el nodo Gateway y los nodos sensor; no obstante, como se mencionó en la métrica de topología, para cubrir mayor extensión, si se desea, es necesario interconectar nodos sensores en una topología de árbol. Por otro lado, el dispositivo Gateway permite opciones de comunicación con el acceso a 
Internet mediante cableado (ethernet) o con el uso de alguna red de telefonía celular 4G, 3G o 2G.

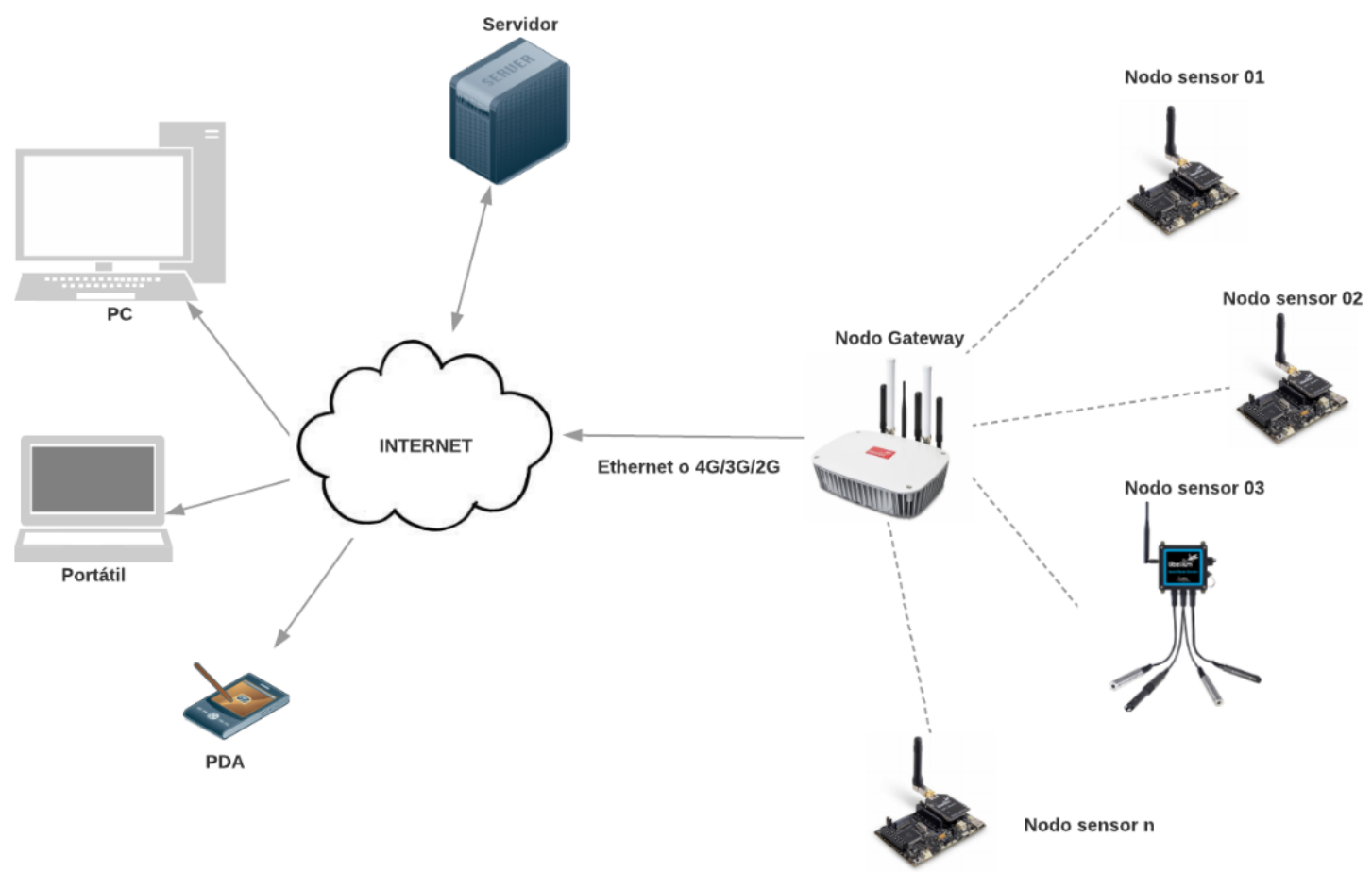

Figura 5. Arquitectura de red del sistema de telemetría. (Fuente: elaboración propia).

La Tabla 3 describe algunas opciones que ofrecen distintos fabricantes.

Tabla 3. Nodos sensores de algunos fabricantes. 
2020, Instituto Mexicano de Tecnología del Agua

Open Access bajo la licencia CCBY-NC-SA 4.0

(https://creativecommons.org/licenses/by-nc-sa/4.0/)

\begin{tabular}{|c|c|c|c|c|c|c|}
\hline Fabricante & Dispositivo & Parámetros & $\begin{array}{c}\text { Frecuencia } \\
\text { de } \\
\text { operación }\end{array}$ & $\begin{array}{l}\text { Alcance } \\
\text { máximo }\end{array}$ & $\begin{array}{l}\text { Suministro } \\
\text { de energía }\end{array}$ & $\begin{array}{c}\text { Autonomía } \\
\text { máxima }\end{array}$ \\
\hline $\begin{array}{l}\text { CSIRO ICT } \\
\text { Center }\end{array}$ & Fleck3 & $\begin{array}{l}\text { Temperatura, } \\
\text { conductividad, } \\
\text { oxígeno } \\
\text { disuelto, pH, } \\
\text { turbidez, } \\
\text { alcalinidad y } \\
\text { SS, entre otros }\end{array}$ & $\begin{array}{l}2.4 \mathrm{GHz}, \\
915 \mathrm{MHz}\end{array}$ & $1500 \mathrm{~m}$ & $\begin{array}{l}\text { Baterías y } \\
\text { paneles } \\
\text { solares }\end{array}$ & - \\
\hline $\begin{array}{l}\text { Crossbow } \\
\text { (MEMSIC } \\
\text { en la } \\
\text { actualidad) }\end{array}$ & eKo & $\begin{array}{l}\text { Temperatura y } \\
\text { humedad del } \\
\text { suelo, } \\
\text { temperatura y } \\
\text { humedad } \\
\text { relativa del } \\
\text { ambiente, } \\
\text { radiación } \\
\text { solar, } \\
\text { precipitación, } \\
\text { presión } \\
\text { barométrica, } \\
\text { velocidad y } \\
\text { dirección del } \\
\text { viento }\end{array}$ & $2.4 \mathrm{GHz}$ & $\begin{array}{l}\text { De } 150 \mathrm{a} \\
3200 \mathrm{~m}\end{array}$ & $\begin{array}{l}\text { Baterías y } \\
\text { paneles } \\
\text { solares }\end{array}$ & $\begin{array}{l}\text { Cinco años, } \\
\text { tres meses } \\
\text { sin sol }\end{array}$ \\
\hline Libelium & $\begin{array}{l}\text { Waspmote } \\
\text { Smart Water }\end{array}$ & $\begin{array}{l}\text { Temperatura, } \\
\text { conductividad, } \\
\text { oxígeno } \\
\text { disuelto, pH, } \\
\text { turbidez, }\end{array}$ & $\begin{array}{l}2.4 \mathrm{GHz}, \\
868 / 900 \\
\mathrm{MHz}\end{array}$ & $\begin{array}{l}7-24 \\
40 \mathrm{~km}\end{array}$ & $\begin{array}{l}\text { Baterías y } \\
\text { paneles } \\
\text { solares }\end{array}$ & Un año \\
\hline
\end{tabular}


2020, Instituto Mexicano de Tecnología del Agua

Tecnología y

Open Access bajo la licencia CCBY-NC-SA 4.0

(https://creativecommons.org/licenses/by-nc-sa/4.0/)

\begin{tabular}{|l|l|l|l|l|l|l|}
\hline & & $\begin{array}{l}\text { alcalinidad y } \\
\text { sólidos } \\
\text { suspendidos, } \\
\text { entre otros }\end{array}$ & & & & \\
\end{tabular}

Dentro de las opciones, la más completa hasta el momento es la solución de Libelium debido a que puede hacer uso de otros sensores en caso de necesitar cambiar el enfoque de la aplicación. Este fabricante ofrece soluciones integrales para la agricultura, salud, domótica, calidad de aire, logística y otras concernientes a la industria. Para el caso de este estudio, el dispositivo Waspmote Smart Water de Libelium permite medir los parámetros seleccionados mediante los sensores presentados (Tabla $4)$.

Tabla 4. Sensores para medición de calidad de agua.

\begin{tabular}{|c|c|c|c|c|c|}
\hline $\begin{array}{c}\text { Nombre } \\
\text { del } \\
\text { sensor }\end{array}$ & Parámetros & Unidad & Rango & Resolución & Exactitud \\
\hline \multirow{4}{*}{$\begin{array}{l}\text { Aqualabo } \\
\text { OPTOD }\end{array}$} & Temperatura & $\begin{array}{l}\text { Grados } \\
\text { Celsius }\end{array}$ & $\begin{array}{c}\text { De } 0.00 a+50.00 \\
{ }^{\circ} \mathrm{C}\end{array}$ & $0.01^{\circ} \mathrm{C}$ & $\pm 0.5^{\circ} \mathrm{C}$ \\
\hline & \multirow{3}{*}{ Oxígeno } & $\begin{array}{c}\% \\
\text { saturación }\end{array}$ & $\begin{array}{c}\text { De } 0.0 \text { a } 200.0 \% \\
\text { SAT }\end{array}$ & 0.1 & $\pm 1 \%$ \\
\hline & & $\mathrm{mg} / \mathrm{l}$ & $\begin{array}{c}\text { De } 0.00 \text { a } 20.00 \\
\mathrm{mg} / \mathrm{l}\end{array}$ & 0.01 & $\pm 0.1 \mathrm{mg} / \mathrm{L}$ \\
\hline & & ppm & $\begin{array}{c}\text { De } 0.00 \text { a } 20.00 \\
\text { ppm }\end{array}$ & 0.01 & $\pm 0.1 \mathrm{ppm}$ \\
\hline
\end{tabular}


Tecnología y

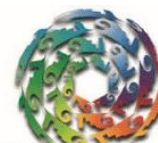

Ciencias $\approx$ Agua
2020, Instituto Mexicano de Tecnología del Agua

Open Access bajo la licencia CCBY-NC-SA 4.0

(https://creativecommons.org/licenses/by-nc-sa/4.0/)

\begin{tabular}{|c|c|c|c|c|c|}
\hline \multirow{3}{*}{$\begin{array}{c}\text { Aqualabo } \\
\text { C4E }\end{array}$} & Temperatura & $\begin{array}{l}\text { Grados } \\
\text { Celsius }\end{array}$ & $\begin{array}{c}\text { De } 0.00 a+50.00 \\
{ }^{\circ} \mathrm{C}\end{array}$ & $0.01{ }^{\circ} \mathrm{C}$ & $\pm 0.5^{\circ} \mathrm{C}$ \\
\hline & Conductividad & $\mu S / c m$ & $\begin{array}{c}\text { Cuatro rangos a } \\
\text { elegir (o } \\
\text { automático): } \\
0-200.0 \mu \mathrm{S} / \mathrm{cm} \\
0-2000 \mu \mathrm{S} / \mathrm{cm} \\
0.00-20.00 \\
\mathrm{mS} / \mathrm{cm} \\
0.0-200.0 \mathrm{mS} / \mathrm{cm} \\
\text { Automático }\end{array}$ & $\begin{array}{c}0.01 \text { a } 1 \\
\text { Según el rango }\end{array}$ & $\begin{array}{c} \pm 1 \% \text { Rango } \\
\text { completo }\end{array}$ \\
\hline & Salinidad & $\mathrm{PPT}=\mathrm{g} / \mathrm{kg}$ & De 5 a $60 \mathrm{~g} / \mathrm{kg}$ & $\begin{array}{c}0.01 \text { a } 1 \\
\text { Según el rango }\end{array}$ & $\begin{array}{c} \pm 1 \% \\
\text { Rango } \\
\text { completo }\end{array}$ \\
\hline \multirow[b]{2}{*}{$\begin{array}{c}\text { Aqualabo } \\
\text { NTU }\end{array}$} & Temperatura & $\begin{array}{l}\text { Grados } \\
\text { Celsius }\end{array}$ & $\begin{array}{c}\text { De } 0.00 a+50.00 \\
{ }^{\circ} \mathrm{C}\end{array}$ & $0.01^{\circ} \mathrm{C}$ & $\pm 0.5^{\circ} \mathrm{C}$ \\
\hline & $\begin{array}{c}\text { Nefelométrico } \\
\text { turbiedad }\end{array}$ & NTU & $\begin{array}{c}\text { De } 0 \text { a } 4000 \text { NTU } \\
\text { Cuatro rangos } \\
\text { para elegir } \\
\text { Parámetros } 1 \text { y } 2 \\
\text { (o } \\
\text { automático): } \\
\text { - Rango 1: } 0 / 50 \\
\text { NTU } \\
\text { (FNU) } \\
\text { - Rango 2: } 0 / 200 \\
\text { NTU } \\
\text { (FNU) } \\
\text { - Rango 3: } 0 / 1000 \\
\text { NTU }\end{array}$ & $\begin{array}{c}0,01 \text { a } 1 \text { NTU - } \\
\mathrm{mg} / \mathrm{L}\end{array}$ & $\begin{array}{c} \pm<5 \% \\
\text { lleno } \\
\text { distancia } \\
\text { Rango 1: } \\
\pm 2.5 \text { NTU } \\
\text { Rango 2: } \\
\pm \text { 10NTU } \\
\text { Rango 3: } \pm \\
50 \\
\text { NTU } \\
\text { Rango 4: }\end{array}$ \\
\hline
\end{tabular}


Tecnología y

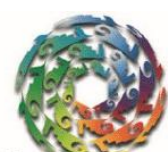

Ciencias đ̊gua
2020, Instituto Mexicano de Tecnología del Agua

Open Access bajo la licencia CC BY-NC-SA 4.0

(https://creativecommons.org/licenses/by-nc-sa/4.0/)

\begin{tabular}{|c|c|c|c|c|c|}
\hline & & & $\begin{array}{c}\text { (FNU) } \\
\text { - Rango 4: 0/4000 } \\
\text { NTU } \\
\text { (FNU) } \\
\text {-Automático }\end{array}$ & & $\begin{array}{l}\text { FNU } \pm 200 \\
\text { NTU }\end{array}$ \\
\hline & & $\begin{array}{l}\text { FNU ( } 1 \text { FNU } \\
=1 \mathrm{NTU})\end{array}$ & $\begin{array}{c}\text { Mismos rangos } \\
\text { que el parámetro } \\
\text { anterior }\end{array}$ & & \\
\hline & $\begin{array}{c}\text { SS (sólidos } \\
\text { suspendidos) }\end{array}$ & $\mathrm{mg} / \mathrm{L}$ & De 0 a $4500 \mathrm{mg} / \mathrm{l}$ & & \\
\hline & Temperatura & $\begin{array}{l}\text { Grados } \\
\text { Celsius }\end{array}$ & $\begin{array}{c}\text { De } 0.00 a+50.00 \\
{ }^{\circ} \mathrm{C}\end{array}$ & $0.01{ }^{\circ} \mathrm{C}$ & $\pm 0.5^{\circ} \mathrm{C}$ \\
\hline PHEHT & $\mathrm{pH}$ & $\mathrm{pH}$ & $\begin{array}{c}\text { De } 0.00 \text { a } 14.00 \\
\mathrm{pH}\end{array}$ & $0.01 \mathrm{pH}$ & $\pm 0.1 \mathrm{pH}$ \\
\hline & $\mathrm{pH}$ & $\mathrm{mV}$ & - & - & - \\
\hline
\end{tabular}

El uso de estos sensores permite abarcar las mediciones necesarias de parámetros de calidad de agua descritos en apartados anteriores. Cada nodo sensor conecta tres sondas (sensores); por ende, si es necesario el uso de los cuatro en un mismo punto, se debe colocar un nodo sensor adicional. 


\section{Conclusiones}

Es un hecho que el uso de la telemetría como herramienta de obtención de parámetros de calidad de agua en tiempo real está empezando a desafiar la forma de realizar este tipo de mediciones en ríos.

La importancia de la telemetría para el monitoreo de los parámetros de calidad de agua propiciará una regulación en el uso actual o futuro del recurso hídrico, garantizando un control y cumplimiento con las legislaciones o políticas nacionales e internacionales.

De igual manera, la telemetría contribuirá a que se mantenga actualizada la información de carácter físico y biótico del agua, permitiendo así evaluar la magnitud de los cambios que resulten en el entorno, en tiempos adecuados para tomar acciones pertinentes.

A través de una revisión de la literatura científica se pudieron determinar tecnologías para la medición de los principales parámetros físico-químicos de agua en ríos que, en conjunto con parámetros de interés para la calidad de agua definidos por la normatividad colombiana, permitieron determinar una propuesta de arquitectura para un sistema de telemetría para el río Magdalena en Barranquilla, Colombia, en que además se propone un listado potencial de nodos sensores basado en 
criterios de conectividad, alcance de transmisión, suministro de energía eléctrica, escalabilidad, topología.

El análisis de la literatura permitió identificar el uso de sensores remotos como una de las principales tecnologías de telemetría, con ésta se identificaron los diferentes sensores utilizados, el sistema de procesamiento de la información y arquitectura de red de los sensores usados en casos de telemetría para calidad de agua en ríos. La solución tecnológica presentada por el fabricante Libelium ofrece las prestaciones necesarias en cuanto a escalabilidad del sistema y compatibilidad con los sensores mencionados en este escrito.

A partir de los resultados del presente estudio, se espera iniciar el despliegue de proyectos de implementación o desarrollo de prototipos de telemetría para la medición de parámetros de calidad de agua sobre el río Magdalena o cualquier otro que se relacione con sus características.

Cabe señalar la importancia de trabajos futuros que comparen los diferentes sistemas de telemetría ofrecidos por los fabricantes para la medición de parámetros de calidad de agua con respecto a la fiabilidad del sistema y confiabilidad de los datos corriendo experimentos y pruebas reales. La ejecución de estas pruebas de rendimiento será preponderante para identificar factores e indicadores del prototipo de medición, proyectando a posteriori el despliegue de un sistema de telemetría para la medición de calidad de agua en ríos.

\section{Agradecimientos}


Los autores desean expresar sus agradecimientos a Elías González Vallejo por la toma de las fotos aéreas para el presente trabajo: río Magdalena (Figura 3) y Puente Pumarejo (Figura 4).

\section{Referencias}

Aguirre, S. E., Piraneque, N. V., \& Cruz, R. K. (2018). Sustancias naturales: alternativa para el tratamiento de agua del río Magdalena en Palermo, Colombia. Información Tecnológica, 29(3), 59-70. Recuperado de https://doi.org/http://dx.doi.org/10.4067/S071807642018000300059

Al-Omari, A., Al-Houri, Z., \& Al-Weshah, R. (2013). Impact of the as samra wastewater treatment plant upgrade on the water quality (COD, electrical conductivity, TP, TN) of the Zarqa River. Water Science and Technology, 67(7), 1455-1464. Recuperado de https://doi.org/10.2166/wst.2013.686

Alahi, E. E., Pereira-Ishak, N., Mukhopadhyay, S., \& Burkitt, L. (2018). An Internet-of-things enabled smart sensing system for nitrate monitoring. IEEE Internet of Things Journal, 5(6), 4662. Recuperado de https://doi.org/10.1109/JIOT.2018.2809669

Alves, R. I. S., Sampaio, C. F., Nadal, M., Schuhmacher, M., Domingo, J. L., \& Segura-Muñoz, S. I. (2014). Metal concentrations in surface water and sediments from Pardo River, Brazil: Human health risks. Environmental Research, 133, 149-155. Recuperado de https://doi.org/10.1016/j.envres.2014.05.012 
Angulo, J. (2017). Barranquilla's water distribution system: A first detailed description. Procedia Engineering, 186(5), 12-19. Recuperado de https://doi.org/10.1016/j.proeng.2017.03.202

Ardila-León, J. F., \& Quintero-Delgado, Ó. Y. (2013). Aplicación de la teledetección y los sistemas de información geográfica en la interpretación de zonas inundables. Caso de estudio: río Soapaga, sector Paz de Río, Boyacá. Ciencia e Ingeniería Neogranadina, 23(2), 55. Recuperado de https://doi.org/10.18359/rcin.223

Barreto, M., Barrera, R., \& Benavides, J. (1999). Una aplicación de sensores remotos y SIG como contribución al manejo integrado de zonas costeras. Punta Rada-Tolú, Colombia: Centro de Investigación y Desarrollo en Información Geográfica (CIAF).

Beatty, S. J., Tweedley, J. R., Cottingham, A., Ryan, T., Williams, J., Lynch, K., \& Morgan, D. L. (2018). Entrapment of an estuarine fish associated with a coastal surge barrier can increase the risk of mass mortalities. Ecological Engineering, 122, 229-240. Recuperado de https://doi.org/10.1016/j.ecoleng.2018.07.009

Caicedo-Carrascal, F. (2008). Asimilación de precipitación estimada por imágenes de satélite en modelos hidrológicos aglutinados y distribuidos, caso de estudio afluencias al embalse de Betania. Huila, Colombia: Pontificia Universidad Javeriana.

Cama-Pinto, A., Acosta-Coll, M., Piñeres-Espitia, G., Caicedo-Ortiz, J., Zamora-Musa, R., \& Sepúlveda-Ojeda, J. (2016). Diseño de una red de sensores inalámbricos para la monitorización de inundaciones repentinas en la ciudad de Barranquilla, Colombia. Ingeniare. Revista 
Ciencias $₫$ Agua
2020, Instituto Mexicano de Tecnología del Agua

Open Access bajo la licencia CC BY-NC-SA 4.0

(https://creativecommons.org/licenses/by-nc-sa/4.0/)

Chilena de Ingeniería, 24(4), 581-599. Recuperado de https://doi.org/10.4067/s0718-33052016000400005

Chen, J., \& Lu, J. (2014). Effects of land use, topography and socioeconomic factors on river water quality in a mountainous watershed with intensive agricultural production in East China. Plos One, 9(8), 1-12. Recuperado de https://doi.org/10.1371/journal.pone.0102714 Damanik-Ambarita, M., Boets, P., Nguyen-Thi, H. T, Eurie-Forio, M. A., Everaert, G., Lock, K., Musonge, P. L. S., Suhareva, N., Bennetsen, E., Gobeyn, S., Ho, T. L., Dominguez-Granda, L., \& Goethals, P. L. M. (2018). Impact assessment of local land use on ecological water quality of the Guayas river basin (Ecuador). Ecological Informatics, 48(August), 226-237. Recuperado de https://doi.org/10.1016/j.ecoinf.2018.08.009

Ding, H., Li, R. R., Lin, H., \& Wang, X. (2016). Monitoring and evaluation on water quality of Hun River based on Landsat satellite data. Recuperado de https://doi.org/10.1109/PIERS.2016.7734699

Forero-Bernal, C. A., Zabala-Parra, P. A., \& Boada-Rodríguez, A. (2017). Análisis espacio-temporal de la incidencia antrópica en la cuenca del río Cauca, en el departamento del Valle. Perspectiva Geográfica, 22(1), 127-146. Recuperado

de https://doi.org/10.19053/01233769.5861

Georgieva, S., Gartsiyanova, K., Ivanova, V., \& Vladimirova, L. (2018). Assessment of physical-chemical characteristics of surface water from key sites of the Mesta River: State and environmental implications. Euroinvent $I C I R$ 2018, 374

Recuperado de 
Tecnología y

Ciencias $₫$ Agua
2020, Instituto Mexicano de Tecnología del Agua

Open Access bajo la licencia CC BY-NC-SA 4.0

(https://creativecommons.org/licenses/by-nc-sa/4.0/)

https://doi.org/10.1088/1757-899X/374/1/012093

Gómez, D. J. L., \& Dalence, M. S. J. (2014). Determinación del parámetro sólidos suspendidos totales (SST) mediante imágenes de sensores ópticos en un tramo de la cuenca media del río Bogotá. Revista UD y La Geomática, (9), 19-27. Recuperado de https://doi.org/http://dx.doi.org/10.14483/udistrital.jour.udgeo.201 4.9.a02

https://revistas.udistrital.edu.co/index.php/UDGeo/article/view/794 3/10942

Google Maps. (s.f., a). Mapa de Barranquilla. Recuperado de https://earth.google.com/web/@10.98381195,-

74.81801753,104.21639152a,22832.34162584d,35y,0h,0t,0r

Google Maps. (s.f., b). Mapa del Departamento de Atlántico. Recuperado de https://earth.google.com/web/@10.98381195,-

74.81801753,104.21639152a,22832.34162584d,35y,0h,0t,0r

Guaman, J., Astudillo-Salinas, F., Vázquez-Rodas, A., Minchala, L. I., \& Placencia, S. (2018). Water level monitoring system based on LoPy4 microcontroller with LoRa technology. Recuperado de https://doi.org/10.1109/INTERCON.2018.8526436

Hatami, R. (2018). Development of a protocol for environmental impact studies using causal modelling. Water Research, 138, 206-223. Recuperado de https://doi.org/10.1016/j.watres.2018.03.034

IDEAM, Instituto de Hidrología, Meteorología y Estudios Ambientales. (2014). Análisis físico químicos de aguas-métodos de análisis. Bogotá, Colombia: Instituto de Hidrología, Meteorología y Estudios 
Tecnología y

Ciencias $₫$ Agua
2020, Instituto Mexicano de Tecnología del Agua

Open Access bajo la licencia CC BY-NC-SA 4.0

(https://creativecommons.org/licenses/by-nc-sa/4.0/)

Ambientales.

Jin, N., Ma, R., Lv, Y., Lou, X., \& Wei, Q. (2010). A novel design of water environment monitoring system based on WSN. Recuperado de https://doi.org/10.1109/ICCDA.2010.5541305

Kamble, P. R., \& Vatti, R. A. (2015). Structural health monitoring of river bridges using wireless sensor. Recuperado de https://doi.org/10.1109/PERVASIVE.2015.7086979

Khan, T. A., Alam, M., Shahid, Z., \& Suud, M. M. (2017). Prior investigation for flash floods and hurricanes, concise capsulization of hydrological technologies and instrumentation: A survey. Recuperado de https://doi.org/10.1109/ICETSS.2017.8324170

Kraus, R. T., Secor, D. H., \& Wingate, R. L. (2015). Testing the thermalniche oxygen-squeeze hypothesis for estuarine striped bass. Environmental Biology of Fishes, 98(10), 2083-2092. Recuperado de https://doi.org/10.1007/s10641-015-0431-3

Liu, L., Chen, L., Gao, P., \& Chen, G. (2011). Study on water quality assessment of urban river. Recuperado de https://doi.org/10.1109/CDCIEM.2011.522

Ma, J., Meng, F., Zhou, Y., Wang, Y., \& Shi, P. (2018). Intelligent water pollution source identification and localization in wireless sensor networks. Recuperado

de https://doi.org/10.1109/IMCEC.2018.8469723

Mahmoud, K. H. (2013). Data collection and processing from distributed system of wireless sensors (tesis de maestría). Masaryk University, Faculty of Informatics, Brno, Chequia. 
Martínez, I. \& Pinilla, G. (2017). Índice de estado limnológico fluvial para los ríos de la cuenca alta del río Chicamocha, Boyacá-Colombia. Revista Luna Azul, 46(46), 125-144. Recuperado de https://doi.org/10.17151/luaz.2018.46.8 https://www.redalyc.org/jatsRepo/3217/321759619008/html/index. html

Mustapha, A., Aris, A. Z., Juahir, H., Ramli, M. F., \& Kura, N. U. (2013). River water quality assessment using environmentric techniques: Case study of Jakara River Basin. Environmental Science and Pollution Research, 20(8), 5630-5644. Recuperado de https://doi.org/10.1007/s11356-013-1542-z

Naddeo, V., Scannapieco, D., Zarra, T., \& Belgiorno, V. (2013). River water quality assessment: Implementation of non-parametric tests for sampling frequency optimization. Land Use Policy, 30(1), 197205. Recuperado

de

https://doi.org/10.1016/j.landusepol.2012.03.013

Oviedo-Machado, N., \& Reinoso-Flórez, G. (2018). Aspectos ecológicos de larvas de Chironomidae (Diptera) del río Opia (Tolima, Colombia). Revista Colombiana de Entomología, 44(1), 101. Recuperado de https://doi.org/10.25100/socolen.v44i1.6546

Panda, U., Sundaray, S., Rath, P., Nayak, B., \& Bhatta, D. (2006). Application of factor and cluster analysis for characterization of river and estuarine water systems. A case study: Mahanadi River (India). Journal of Hydrology, 331(3-4), 434-445. Recuperado de https://doi.org/10.1016/j.jhydrol.2006.05.029 
Pant, D., Verma, S., \& Dhuliya, P. (2017). A study on disaster detection and management using WSN in Himalayan region of Uttarakhand. Recuperado de https://doi.org/10.1109/ICACCAF.2017.8344703

Pérez, J. I., Nardini, G. A., \& Galindo, A. A. (2018). Comparative analysis of water quality indices applied to the Ranchería River, La GuajiraColombia. Información Tecnológica, 29(3), 47-58. Recuperado de https://doi.org/http://dx.doi.org/10.4067/S071807642018000300047

Prafanto, A., \& Budiman, E. (2018). A Water Level Detection: IoT Platform Based on Wireless Sensor Network. Proceedings - 2nd East Indonesia Conference on Computer and Information Technology: Internet of Things for Industry, EIConCIT 2018, 46-49. https://doi.org/10.1109/EIConCIT.2018.8878559

Raimondi, F. M., Trapanese, M., Franzitta, V., \& Viola, A. (2015). A innovative monitoring underwaterbuoy systems (MUnBuS) for marine and rivers installation with IR-Cam, instrumental telemetry and acoustic data acquisition Capability. Recuperado de https://doi.org/10.1109/OCEANS-Genova.2015.7271600

Ramírez-Cerpa, E., Acosta-Coll, M., \& Vélez-Zapata, J. (2017). Análisis de condiciones climatológicas de precipitaciones de corto plazo en zonas urbanas: caso de estudio Barranquilla, Colombia. Idesia (Arica), 35(2).

Recuperado

de

https://doi.org/http://dx.doi.org/10.4067/S0718-

34292017005000023

Restrepo, J. C., Schrottke, K., Traini, C., Ortíz, J. C., Orejarena, A., Otero, L., \& Marriaga, L. (2015). Sediment transport and geomorphological 
Tecnología y

Ciencias Agua
2020, Instituto Mexicano de Tecnología del Agua

Open Access bajo la licencia CC BY-NC-SA 4.0

(https://creativecommons.org/licenses/by-nc-sa/4.0/)

change in a high-discharge tropical delta (Magdalena River, Colombia): Insights from a period of intense change and human intervention (1990-2010). Journal of Coastal Research, 319, 575589. Recuperado de https://doi.org/10.2112/jcoastres-d-14-00263.1

Samijayani, O. N., Sulistya, F. G., Wulansari, M. T., Mujadin, A., \& Rahmatia, S. (2018). Wireless sensor network for $\mathrm{pH}$ and turbidity of river water monitoring. Recuperado de https://doi.org/10.1063/1.5080038

Sánchez-Comas, A., Neira, D., \& Cabello, J. J. (2016). Marcos aplicados a la gestión de calidad. Una revisión sistemática de la literatura. Espacios, 37(9), 17.

Sfikas, A., Angelidis, P., \& Petridis, D. (2015). A statistical approach for identification of potential pollution incidents due to lignite mining activity in a surface water stream. Desalination and Water Treatment, 57(40), 18606-18618. Recuperado de https://doi.org/10.1080/19443994.2015.1100559

Shen, S., Sun, L., Dang, Y., Zou, Z., \& Wang, R. (2018). Node localization based on improved PSO and mobile nodes for environmental monitoring WSNs. International Journal of Wireless Information Networks, 25(4), 470-479. Recuperado de https://doi.org/10.1007/s10776-018-0414-3

Shetty, S., Pai, R. M., \& Pai, M. M. M. M. (2018). Design and implementation of aquaculture resource planning using underwater sensor wireless network. Cogent Engineering, 5(1), 1-23. Recuperado de https://doi.org/10.1080/23311916.2018.1542576 
Singh, D. K., Gusain, H. S., Mishra, V., Gupta, N., \& Das, R. K. (2018). Automated mapping of snow/ice surface temperature using Landsat8 data in Beas River Basin, India, and validation with wireless sensor network data. Arabian Journal of Geosciences, 11(6). Recuperado de https://doi.org/10.1007/s12517-018-3497-3

Tavakoly, S. S. B., Monazami, G., Rezayi, M., Tajfard, M., \& Borgheipour, H. (2018). Application of water quality indices for evaluating water quality and anthropogenic impact assessment. International Journal of Environmental Science and Technology, 16, 3001-3012. Recuperado de https://doi.org/10.1007/s13762-018-1894-5

Tétard, S., Feunteun, E., Bultel, E., Gadais, R., Bégout, M. L., Trancart, T., \& Lasne, E. (2016). Poor oxic conditions in a large estuary reduce connectivity from marine to freshwater habitats of a diadromous fish. Estuarine, Coastal and Shelf Science, 169, 216-226. Recuperado de https://doi.org/10.1016/j.ecss.2015.12.010

Torres, B. F., Ramírez-León, H., Rodríguez, C. C., Tejera, G. M. P., \& Vásquez, J. M. C (2015). Validación de un modelo hidrodinámico y calidad del agua para el río Magdalena, en el tramo adyacente a Barranquilla, Colombia. Hidrobiológica, 25(1), 7-23. Recuperado de https://doi.org/10.1111/j.1750-2659.2010.00186.x

Turnure, J. T., Grothues, T. M., \& Able, K. W. (2014). Seasonal residency of adult weakfish (Cynoscion regalis) in a small temperate estuary based on acoustic telemetry: A local perspective of a coast wide phenomenon. Environmental Biology of Fishes, 98(5), 1207-1221. Recuperado de https://doi.org/10.1007/s10641-014-0353-5 
Velásquez-Villada, C., \& Donoso, Y. (2016). Delay/disruption tolerant network-based message forwarding for a river pollution monitoring wireless sensor network application. Sensors, 16(4), 436. Recuperado de https://doi.org/10.3390/s16040436

Vilardy, S. P. (2015). Dinámicas complejas del río Magdalena: necesidad de un marco integral de gestión de la resiliencia ante el cambio climático. En: Becerra, R. M. (ed.). ¿Para dónde va el Río Magdalena? Riesgos sociales, ambientales y económicos del proyecto de navegabilidad (pp. 1-15). Bogotá, Colombia: Friedrich-Ebert-Stiftung en Colombia (Fescol).

Viswanathan, A., Sai Shibu, N. B., Rao, S. N., \& Ramesh, M. V. (2017). Security challenges in the integration of IoT with WSN for smart grid applications. Recuperado

de https://doi.org/10.1109/ICCIC.2017.8524233

Zhou, Q., Chen, C., Zhang, G., Chen, H., Chen, D., Yan, Y., Shen, J., \& Zhou, R. (2018). Real-time management of groundwater resources based on wireless sensors networks. Journal of Sensor and Actuator Networks, 7(1), 4. Recuperado de https://doi.org/10.3390/jsan7010004 